Article

\title{
Glycerolipid Composition and Advanced Physicochemical Considerations of Sacha Inchi Oil toward Cosmetic Products Formulation
}

\author{
Diana Penagos-Calvete ${ }^{1}$, Valeria Duque ${ }^{1}$, Claudia Marimon ${ }^{1}$, Diana M. Parra ${ }^{2}$, \\ Sandra K. Restrepo-Arango ${ }^{2}$, Oliver Scherf-Clavel ${ }^{3}{ }^{\mathbb{D}}$, Ulrike Holzgrabe $^{3}{ }^{\mathbb{D}}$, \\ Guillermo Montoya ${ }^{2, *(D)}$ and Constain H. Salamanca 1,2,*(D) \\ 1 Maestría en Formulación de Productos Químicos y Derivados, Facultad de Ciencias Naturales, \\ Universidad Icesi, Calle 18 No. 122-135, Cali 76003, Colombia; dpenagos@icesi.edu.co (D.P.-C.); \\ valeduke9@hotmail.com (V.D.); claudiapmj@gmail.com (C.M.) \\ 2 Departamento de Ciencias Farmacéuticas, Facultad de Ciencias Naturales, Universidad Icesi, Cali 76003, \\ Colombia; dianaparra-2006@hotmail.com (D.M.P.); karirestrepo21@gmail.com (S.K.R.-A.) \\ 3 Institute for Pharmacy and Food Chemistry, University of Würzburg, 97074 Würzburg, Germany; \\ oliver.scherf-clavel@uni-wuerzburg.de (O.S.-C.); ulrike.holzgrabe@uni-wuerzburg.de (U.H.) \\ * Correspondence: glmontoya@icesi.edu.co (G.M.); chsalamanca@icesi.edu.co (C.H.S.)
}

Received: 1 November 2019; Accepted: 6 December 2019; Published: 9 December 2019

\begin{abstract}
Sacha inchi oil is a premier raw material with highly nutritional and functional features for the foodstuff, pharmaceutical, beauty, and personal care industries. One of the most important facts about this oil is the huge chemical content of unsaturated and polyunsaturated fatty acids. However, the current available information on the characterization of the triglyceride composition and the advance physicochemical parameters relevant to emulsion development is limited. Therefore, this research focused on providing a detailed description of the lipid composition using high-resolution tandem mass spectrometry and thorough physicochemical characterization to find the value of the required hydrophilic-lipophilic balance (HLB). For this, a study in the interfacial tension was evaluated, followed by the assessment of different parameters such as creaming index, droplet size, viscosity, zeta potential, $\mathrm{pH}$, and electrical conductivity for a series emulsified at thermal stress condition. The results show that fatty acids are arranged into glycerolipids and the required HLB to achieve the maximum physical stability is around 8 .
\end{abstract}

Keywords: sacha inchi oil; unsaturated fatty acids; triacylglycerides; high-resolution tandem mass spectrometry; emulsions oil-in-water; required hydrophilic-lipophilic balance

\section{Introduction}

Glycerolipids are the most important source of energy storage in higher eukaryotes and almost all plant oils are derived from seeds or seed mesocarp-like tissues. The consumption of edible vegetal oil is expected to double over the coming decades and, more importantly, oils performing a double role as food ingredient, and exotic-functional constituent in cosmetic formulations. There is an increasing interest in sacha inchi oil (SIO), and its industrialization has apparently supplied the market needs, and new and improved methodologies are being developed to better extract yields and preserve the highest nutritional capacity [1]. Plukenetia volubilis L. production has expanded throughout the Andean region, and some researchers are interested in finding more Plukenetia species which can be more robust and produce bigger seeds [2]. At the same time, this crop plays a crucial role in the social conflict in Colombia, where it has been used as a voluntary crop substitution for illegal crops, which have been forcefully eradicated. 
The conditions of the Andean Amazon expanse are shared between Brazil, Peru, Ecuador, and Colombia, and the Sacha inchi crop is rapidly growing due to these similar soil conditions - specifically pluviosity and light cycles. Sacha inchi production in Colombia is carried out in the states of Putumayo, Cauca, Nariño, and Caquetá, but it is also grown elsewhere due to government initiatives and constitutes one of the new and prominent agricultural products.

Regarding its nutritional properties, SIO is tremendously high in polyunsaturated fatty acids such as alpha linoleic acid (18:3) and linoleic acid (18:2), both constituting between 80 and $85 \%$ of the fatty acid content. SIO also contains other unsaturated fatty acids, such as oleic acid (18:1), and even saturated ones like palmitic acid (16:0) and stearic acid (18:0) [3]. This premier oil may have unique nutritional properties compared to other vegetal oils, and therefore, it has been projected as a new leading ingredient for advanced formulations in the cosmetics, pharmaceutical, and food industries. Additionally, its glycerolipid content has also been studied in order to understand its physical, chemical, and functional properties [4].

Regarding the foodstuff industry, SIO could be used as a nutritional alternative equivalent to other types of vegetables oils but with higher nutritional and commercial values. SIO can be used combined with olive oil [5], coconut oil [6], argan oil [7], and avocado oil [8]. For emulsify products development, containing active pharmacological ingredients prone to oxidation, the SIO would be incorporated providing an extra protective effect [9]. On the other hand, in the beauty and personal care industry, this oil provides additional types of cosmetic properties. For instance, the SIO could be employed as a "natural raw material" [10-12], as an "antioxidant ingredient" [13] or, due to its origin, as an "exotic oil" [12].

A handful of studies have reported the chemical composition of SIO $[3,14,15]$, but only a few of them have worked with the physicochemical characterization throughout heterodisperse formulations [16-20]. For this reason, this research has focused on exploring two specific aspects of SIO, corresponding to (i) the detailed characterization of glycerolipids composition, and (ii) the determination of the required hydrophilic-lipophilic balance value (HLB) [21,22]. The HLB value is an important physicochemical parameter commonly used in the development of oil-in-water type emulsions.

\section{Materials and Methods}

\subsection{Sacha Inchi Oil Purchasing}

The Sacha inchi (Plukenetia volubilis L.) oil is commercially obtained from a small agricultural cooperative located in the Municipality of Santander de Quilichao, (Cauca-Colombia). The industrial extraction process comprises mechanical seed pressing (cold pressing) and correct storage conditioning, which preserves oil oxidation until commercialization. The company provided twenty liters of extra virgin oil for the experimental work.

\subsection{Chemical and Reagents}

The hypergrade methanol, acetonitrile, and acetone for LC-MS were purchased from Sigma-Aldrich (St Louis, Missouri, USA). Ingredients used in emulsified formulations were steareth 2 (Brij ${ }^{\mathrm{TM}} \mathrm{S} 2$, $\mathrm{HLB}=4.9$, melting point $\left.=42^{\circ} \mathrm{C}-46^{\circ} \mathrm{C}\right)$, steareth $20\left(\right.$ Brij ${ }^{\mathrm{TM}} \mathrm{S} 20, \mathrm{HLB}=15.3$, melting point $\left.=56^{\circ} \mathrm{C}-60^{\circ} \mathrm{C}\right)$, glyceryl stearate (Cithrol ${ }^{\mathrm{TM}} \mathrm{GMS}, \mathrm{HLB}=3.8$, melting point $=57^{\circ} \mathrm{C}-60^{\circ} \mathrm{C}$ ) and polyoxyl 40 stearate $\left(\right.$ Myrj $^{\mathrm{TM}} \mathrm{S} 40, \mathrm{HLB}=17.5$, melting point $\left.=44^{\circ} \mathrm{C}-47^{\circ} \mathrm{C}\right)$, sorbitan oleate $\left(\right.$ Span $^{\mathrm{TM}} 80, \mathrm{HLB}=4.3$, melting point $=10^{\circ} \mathrm{C}-12{ }^{\circ} \mathrm{C}$ ) and polysorbate 80 (Tween ${ }^{\mathrm{TM}} 80, \mathrm{HLB}=15$, melting point $=-21^{\circ} \mathrm{C}$ ) purchased at CRODA (Snaith, UK). Methylparaben and propylparaben were acquired from Sigma-Aldrich (St. Louis, MO, USA). Ultrapure water for chromatographic purposes was obtained from an Arium ${ }^{\circledR}$ pro-VF (Sartorius Stedim) with a resistivity of $15 \mathrm{M} \Omega$. cm (at $25^{\circ} \mathrm{C}$ ). 


\subsection{Physicochemical Quality Control and Fatty Acid Methyl Ester Profiles of SIO}

The physicochemical characterization and analysis of the fatty acid methyl ester profiles of SIO were carried out according to the official methods of the American oil chemists' society (AOCS) [23] and United Stated pharmacopoeia (USP) [24]. Analysis of the refractive index, saponification value, peroxide index, iodine value, and acid index were carried out according to guidelines AOCS CC 7-25, AOCS Cd 3-25, AOCS Cd 8-53, AOCS Cd 1c-85, and USP $40<401>$, respectively. At the same time, determination of the fatty acid methyl ester profiles was carried out according to guideline AOCS Ce 1-62. Concerning refractive index, a refractometer Vee Gee C10 was used. The analysis of fatty acid employed a gas chromatograph (Hewlett Packard HP 5890, Series II, Palo Alto, CA, USA) equipped with a flame ionization detector and a BPX70-ms capillary column $(30 \mathrm{~m} \times 0.25 \mathrm{~mm}$ $\times 0.25 \mu \mathrm{m}$ ) composed of $70 \%$ cyanopropylpolysilphenylene-siloxane. The initial temperature was $150{ }^{\circ} \mathrm{C} / \mathrm{min}$, which increased by $5^{\circ} \mathrm{C} / \mathrm{min}$ up to $240^{\circ} \mathrm{C}$. The injector temperature was $240{ }^{\circ} \mathrm{C}$ and the detector temperature was $280^{\circ} \mathrm{C}$, with a split ratio of 1:30. The carrier gas used was He at $1 \mathrm{~mL} / \mathrm{min}$, at a pressure of $11 \mathrm{psi}$.

\subsection{Chromatographic and Mass Spectrometry Conditions}

The UHPLC system consisted of an UltiMate 3000 thermo-scientific, equipped with a quaternary pump, degasser, and preheater modules. For the chromatographic method, a Develosil $3 \mu \mathrm{m}$ RP-Aqueous C30 $150 \times 2$ mm, $140 \AA$ pore size column was used in a $105 \mathrm{~min}$ run. The mobile phases consisted of acetonitrile/water $(80 / 20 \mathrm{v} / \mathrm{v})$ as eluent $A$, and methanol/acetone $(60 / 40 \mathrm{v} / \mathrm{v})$ as eluent $B$ using a gradient program as follows: $0 \min 70 \%$ of $\mathrm{A} ; 10 \min 60 \% \mathrm{~A} ; 15 \min 50 \% \mathrm{~A} ; 20 \min 45 \% \mathrm{~A}, 30$ $\min 40 \% \mathrm{~A} ; 45 \min 35 \% \mathrm{~A} ; 55 \min 30 \% \mathrm{~A} ; 65 \min 20 \% \mathrm{~A} ; 70 \min 10 \% \mathrm{~A} ; 85 \min 5 \% \mathrm{~A} ; 90 \min 1 \% \mathrm{~A}$, $95 \mathrm{~min} 1 \% \mathrm{~A} ; 100 \mathrm{~min} 70 \% \mathrm{~A}, 105 \mathrm{~min} 70 \%$ A. Flow rate was established at $0.4 \mathrm{~mL} \mathrm{~min}^{-1}$. The data analysis was carried out using MestRec Nova 11.0. The chromatographic system was coupled to a Q-Exactive hybrid quadrupole-Orbitrap mass spectrometer, using atmospheric pressure chemical ionization ion source in positive mode. The mass spectrometer conditions were a discharge voltage of $2.48 \mathrm{kV}$, a corona current of $3.50 \mu \mathrm{A}$, a capillary temperature of $270^{\circ} \mathrm{C}$, and a vaporizer temperature of $360^{\circ} \mathrm{C}$. Sample preparation for UHPLC-MS analysis was carried out by diluting the sacha inchi oil in the mobile phase B (methanol/acetone $60 / 40 \mathrm{v} / \mathrm{v}$ ), to a concentration close to $10 \mu \mathrm{g} / \mathrm{mL}$.

\subsection{Determination of the Required Hydrophilic-Lipophilic Balance Value}

Determination of the required HLB value for the SIO was carried out evaluating several methodologies corresponding to interfacial tension, creaming index, drop size, viscosity, zeta potential, $\mathrm{pH}$, and conductivity measurements. For this, several heterodisperse formulations were prepared using SIO, ultrapure water, preservatives (methylparaben and propylparaben), and binary mixtures of different type of surfactants at $2 \% \mathrm{w} / \mathrm{w}$, which were combined in different proportions to provide HLB values of blend $\left(\mathrm{HLB}^{\mathrm{B}}\right)$ of $6,8,10$, and 12 obtained with different mixtures of surfactants (system 1: steareth 2 and steareth 20, system 2: glyceryl stearate and polyoxyl 40 stearate, system 3: Sorbitan oleate and Polysorbate 80). The complete formulations are shown in Supplementary Materials. Each emulsified system was prepared in triplicate.

The values of the HLB surfactants blend were determined according to:

$$
H L B^{B}=H L B_{a} \times \%\left(S_{a}\right)+H L B_{b} \times \%\left(S_{b}\right)
$$

where $\mathrm{HLB}^{\mathrm{B}}$ is the value of the binary surfactant blend, and $\mathrm{HLB}_{\mathrm{a}}$ and $\mathrm{HLB}_{\mathrm{b}}$ are the values of HLB of the respective surfactants used according to the technical sheets. \% $\left(\mathrm{S}_{\mathrm{a}}\right)$ and $\%\left(\mathrm{~S}_{\mathrm{b}}\right)$ is the amount of surfactant mixed considering a base of $100 \% \mathrm{w} / \mathrm{w}$, with respect to such binary blend. 


\subsubsection{Emulsion Preparation Method}

The emulsions were prepared in several stages according to the formulation shown in support material file. First, the SIO and ultrapure water were heated to $60{ }^{\circ} \mathrm{C}$ and $62{ }^{\circ} \mathrm{C}$, respectively. Once the phase temperature was reached, the preservatives were added to the ultrapure water (aqueous phase), while the respective binary surfactant blends (steareth 2-steareth 20, glyceryl stearate-polyoxyl 40 stearate, and poysorbate 80-sorbitan oleate) were added into SIO (oily phase). Afterward, the oily phase was poured into the aqueous phase, using an Ultra-Turrax at $5000 \mathrm{rpm}$ for $10 \mathrm{~min}$. Subsequently, the system was cooled to room temperature, so each emulsified system was ready for further physicochemical characterization studies and thermal stability assays.

\subsubsection{Interfacial Tension Measurements}

The interfacial tension given between a droplet of SIO immersed in ultrapure water was determined using the pendant drop method [25]. For this, we employed the optical contact angle measuring and contour analysis systems OCA15EC from Dataphysics (Software SCA22 version 4.5.14), coupled with a needle SNP 165/119. Furthermore, this experiment was carried out in the absence and presence of surfactants, where polysorbate 80 was solubilized in ultrapure water, while sorbitan oleate was solubilized in SIO. Each measurement was performed in triplicate at approximately $22 \pm 1{ }^{\circ} \mathrm{C}$ and $60 \% \pm 5 \%$ relative humidity.

\subsubsection{Creaming Index Determination}

In this case, the freshly made emulsions were filled in Falcon ${ }^{\mathrm{TM}} 15 \mathrm{~mL}$ conical centrifuge tubes (diameter $=1.5 \mathrm{~cm}$ ) and centrifuged at $3000 \mathrm{rmp}(150 \mathrm{RFC})$ for $4 \mathrm{~h}$ in a Wincon 80-2 centrifuge (Changsha, China). Finally, the value of the creaming index (CI) was determined according to:

$$
C I=\frac{H_{S}}{H_{E}} \times 100
$$

where $\mathrm{H}_{\mathrm{S}}$ is the sediment height and $\mathrm{H}_{\mathrm{E}}$ is the sample height before centrifugation.

\subsubsection{Droplet Size Analysis}

The droplet size distribution of the emulsions was obtained using a Mastersizer 3000 (Malvern Instruments, Worcestershire, UK), equipped with a helium/neon laser at a wavelength of $632.8 \mathrm{~nm}$. Previously, $\sim 0.6 \mathrm{~g}$ of the emulsion was diluted with $10 \mathrm{~mL}$ of ultrapure water at $25 \pm 2{ }^{\circ} \mathrm{C}$ and stirred at $400 \mathrm{rpm}$. The appropriate amount sample was determined, when an obscurance level between $2 \%$ and $8 \%$ was reached in the equipment. Drop size data were expressed as $\mathrm{D}_{[4,3]}[26-28]$ and all the measurements were conducted in triplicate.

\subsubsection{Viscosity Measurement}

Viscosity was measured using a viscometer (micro-visc, RheoSense Inc., San Ramon, CA, USA), applying different shear stress (see support material file). All measurements were performed in triplicate.

\subsubsection{Zeta Potential, $\mathrm{pH}$, and Electrical Conductivity Measurements}

Zeta potential measurements were carried out using a Zetasizer nano ZSP (Malvern Instruments, Worcestershire, UK) at $25 \pm 2{ }^{\circ} \mathrm{C}$ temperature, with equilibration times of $120 \mathrm{~s}$ in a DTS 1070 capillary cell. For these experiments, the attenuator position and intensity were set automatically. To prepare the sample, $130 \mathrm{mg}$ of the emulsified system was diluted in $20 \mathrm{~mL}$ of ultrapure water and stirred manually. From this, a $50 \mu \mathrm{L}$ aliquot was taken and diluted with $1 \mathrm{~mL}$ of ultrapure water before each zeta potential measurement. On the other hand, the electrical conductivity and $\mathrm{pH}$ of the emulsions was determined 
using a CR-30 conductivity meter and a Starter- $2100 \mathrm{pH}$ meter, respectively. All measurements were carried out in triplicate.

\subsection{Thermal Stability Assay of Sacha Inchi Emulsion}

Once the required HLB value of SIO was found, several oil-in-water emulsions were manufactured in triplicate according to the formulation shown in Supplementary Materials, and the method is described in Section 2.5.1. Each emulsion was placed in Falcon ${ }^{\mathrm{TM}} 15 \mathrm{~mL}$ conical centrifuge tubes, which were subsequently left under two temperature conditions $\left(40 \pm 2{ }^{\circ} \mathrm{C}\right.$ and $\left.4.0 \pm 0.5^{\circ} \mathrm{C}\right)$. For this, each thermal condition was varied every week, starting at $40{ }^{\circ} \mathrm{C}$ in the first week, then at $4{ }^{\circ} \mathrm{C}$ and so on until reaching the last week. The stability parameters evaluated were $\mathrm{CI}$, droplet size, viscosity, zeta potential, $\mathrm{pH}$, and electrical conductivity, which were measured under the same conditions previously described in the section about determining the required HLB.

\section{Results and Discussion}

\subsection{Structural Analysis of Glycerolipids By Mass Spectrometry}

Previous reports have focused attention on the fatty acid compositional analysis of SIO [3,4]. They assigned them with the number of carbons followed by the number of double bonds. Linolenic acid, for instance, has three double bonds $(\Delta 9,12,15)$ and is designated as 18:3. Interestingly, the total pattern of glycerolipids in this previous report turns out to be quite similar to our outcomes. We additionally included analysis under high-resolution and tandem mass measurements of the molecular weight and deduced the fatty acid composition using two abundant fragmentations, a neutral loss of each fatty acid, and a specific acyl chain loss that supports the fatty acid composition. These two types of fragmentation paths are explained in Figure 1A-C. $\beta$-cleavage is favored and generates a neutral loss of a fatty acid and the formation of a dioxolane linked to the acyl group [29]. The size of the acyl chain depends on the type of fatty acid bonded. The ionization and fragmentation for glycerolipids are favored in atmospheric pressure chemical ionization (APCI) rather than Electrospray ionization (ESI), which give less informative fragments due their neutrality conditions. In order to obtain more structural information by fragmentation, a more energetic mechanism is reached by APCI [30,31]. $\mathrm{A} \mathrm{MS}_{2}$ tandem mass analysis was carried out and the information at the higher collision energy of each compound is reported in Supplementary Materials.

The acyl chains lost were 184, 206, 208, 210, and 212 corresponding to 256 (palmitic acid 16:0), 278 (linolenic acid 18:3), 280 (linoleic acid 18:2), 282 (oleic acid 18:1), and 284 (stearic acid 18:0). The errors in ppm of the glycerolipid (tri and diglycerides) were down $2 \mathrm{ppm}$, and the neutral fatty acid lost error were under $3 \mathrm{ppm}$ except for compound number 7 (maximum error of $6.7 \mathrm{ppm}$ ). The acyl chains lost are explained in Figure 1C showing the fragmentation of TAG10 from Table 1. A complete list of di and triacylglyceroid content are summarized in Table 1 . The most abundant compounds were TAG13 with a relative abundance of $7 \%(18: 3,18: 1,18: 3)$, TAG14 with $14.7 \%(18: 2,18: 3,18: 2)$, TAG15 with 19.7\% (18:3,18:2,18:3), and TAG16 with 12.1\% (18:3,18:3,18:3). Diacylglycerides DAG17, 18, and 19 are present in relative low abundance in SIO, but also this molecule would be related to a TGA hydrolysis. Our results are in accordance with previous reports regarding chemical profile [4]. 


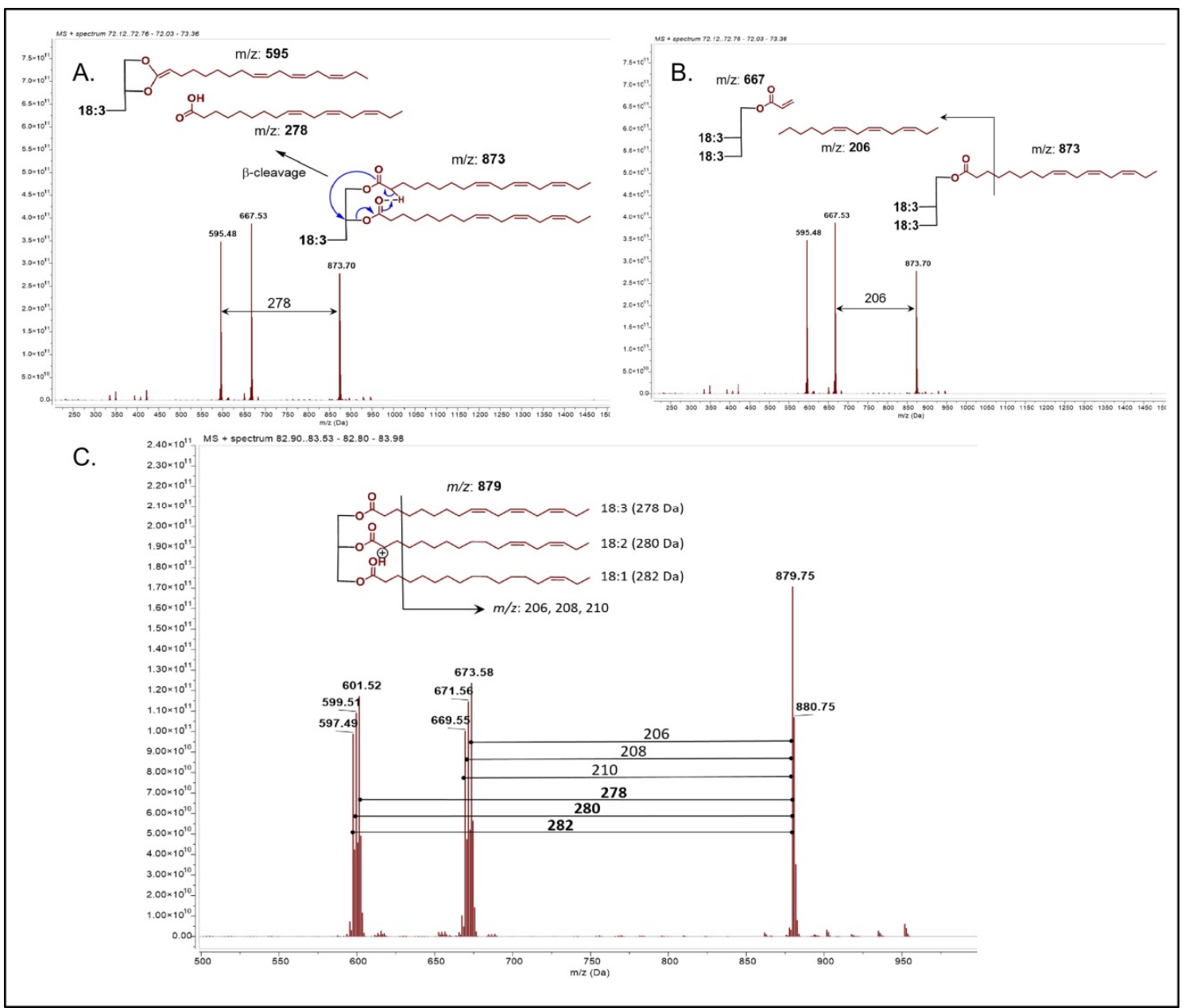

Figure 1. Mass spectrum of TAG 16 (18:3, 18:3, 18:3). (A), the fragmentation mechanism explaining the $\beta$-cleavage and the neutral loss of one fatty acid. (B), the acyl chain loss of 206 corresponding to an 18:3 fatty acid. (C), mass spectrum of TAG (18:3, 18:2, 18:1). Different fatty acids linked to glycerol backbone are demonstrated by the three fatty acid losses with their lost acyl chain, respectively. 
Table 1. A complete glycerolipid profile under high-resolution mass spectrometry. The reported errors were calculated for each compound, and the neutral fatty acid lost with its error were inferred by subtraction.

\begin{tabular}{|c|c|c|c|c|c|c|c|c|c|c|c|c|c|c|}
\hline & & \multirow[b]{2}{*}{$\begin{array}{c}\text { Molecular } \\
\text { Formula }\end{array}$} & \multirow[b]{2}{*}{ Exact Mass } & \multicolumn{2}{|c|}{ Glycerolipid } & \multirow[b]{2}{*}{$\begin{array}{l}\text { Experimental } \\
m / z[\mathrm{M}+\mathrm{H}]^{+}\end{array}$} & \multirow[b]{2}{*}{ Error (ppm) } & \multicolumn{5}{|c|}{ Fatty Acid Composition } & \multirow[b]{2}{*}{$\begin{array}{c}\text { Neutral Acyl } \\
\text { Moiety Losses }\end{array}$} & \\
\hline & & & & $\begin{array}{c}\text { Retention } \\
\text { Time }\end{array}$ & $\begin{array}{l}\text { Theorical m/z } \\
{[M+H]^{+}}\end{array}$ & & & $\begin{array}{l}\text { Neutral Fatty } \\
\text { Acid Looses }\end{array}$ & Theorical $\mathrm{m} / \mathrm{z}$ & $\begin{array}{l}\text { Experimental } \\
m / z[\mathrm{M}+\mathrm{H}]^{+}\end{array}$ & Error (ppm) & Type of Fatty Acid & & \\
\hline 1 & 18:0,18:2,18:2 & $\mathrm{C}_{57} \mathrm{H}_{102} \mathrm{O}_{6}$ & 882.76764 & 98.08 & 883.77492 & 883.77509 & -0.192 & $\begin{array}{l}603.53524 \\
599.50413\end{array}$ & $\begin{array}{l}280.24023 \\
284.27153\end{array}$ & $\begin{array}{l}280.23985 \\
284.27096\end{array}$ & $\begin{array}{l}1.356 \\
2.005\end{array}$ & $\begin{array}{l}\text { Linoleic acid (18:2) } \\
\text { Stearic acid (18:0) }\end{array}$ & $\begin{array}{l}675.58824 \\
671.55205\end{array}$ & $\begin{array}{l}208.1869 \\
212.2230\end{array}$ \\
\hline 2 & $18: 2,18: 1,16: 0$ & $\mathrm{C}_{55} \mathrm{H}_{100} \mathrm{O}_{6}$ & 856.75199 & 96.41 & 857.75927 & 857.75949 & -0.256 & $\begin{array}{l}601.51960 \\
577.51952 \\
575.50431\end{array}$ & $\begin{array}{l}256.24023 \\
280.24023 \\
282.25588\end{array}$ & $\begin{array}{l}256.23989 \\
280.23997 \\
282.25518\end{array}$ & $\begin{array}{l}1.327 \\
0.928 \\
2.480\end{array}$ & $\begin{array}{c}\text { Palmitic acid }(16: 0) \\
\text { Linoleic acid }(18: 2) \\
\text { Oleic acid }(18: 1)\end{array}$ & $\begin{array}{l}673.69391 \\
64961682 \\
647.61991\end{array}$ & $\begin{array}{l}184.0656 \\
208.1427 \\
210.1396\end{array}$ \\
\hline 3 & 18:1,18:2,18:1 & $\mathrm{C}_{57} \mathrm{H}_{102} \mathrm{O}_{6}$ & 882.76764 & 95.17 & 883.77492 & 883.77524 & -0.362 & $\begin{array}{l}601.52011 \\
603.53434\end{array}$ & $\begin{array}{l}282.25588 \\
280.24023\end{array}$ & $\begin{array}{l}282.25513 \\
280.24090\end{array}$ & $\begin{array}{l}2.657 \\
-2.391\end{array}$ & $\begin{array}{c}\text { Oleic acid (18:1) } \\
\text { Linoleic acid (18:2) }\end{array}$ & $\begin{array}{l}673.58455 \\
675.64272\end{array}$ & $\begin{array}{l}210.1907 \\
208.1325\end{array}$ \\
\hline 4 & 18:0,18:2,18:3 & $\mathrm{C}_{57} \mathrm{H}_{100} \mathrm{O}_{6}$ & 880.75199 & 94.22 & 881.75927 & 881.75921 & 0.068 & $\begin{array}{l}603.53497 \\
597.48834 \\
601.51945\end{array}$ & $\begin{array}{l}278.22458 \\
284.27153 \\
280.24023\end{array}$ & $\begin{array}{l}278.22424 \\
284.27087 \\
280.23976\end{array}$ & $\begin{array}{l}1.222 \\
2.322 \\
1.677\end{array}$ & $\begin{array}{l}\text { Linolenic acid (18:3) } \\
\text { Stearic acid (18:0) } \\
\text { Linoleic acid (18:2) }\end{array}$ & $\begin{array}{l}675.59253 \\
669.54589 \\
673.59881\end{array}$ & $\begin{array}{l}206.1667 \\
212.2133 \\
208.1604\end{array}$ \\
\hline 5 & $16: 0,18: 1,18: 3$ & $\mathrm{C}_{55} \mathrm{H}_{98} \mathrm{O}_{6}$ & 854.73634 & 92.82 & 855.74362 & 855.74437 & -0.876 & $\begin{array}{l}599.50428 \\
577.52001 \\
573.48895\end{array}$ & $\begin{array}{l}256.24023 \\
278.22458 \\
282.25588\end{array}$ & $\begin{array}{l}256.24009 \\
278.22436 \\
282.25542\end{array}$ & $\begin{array}{l}0.546 \\
0.791 \\
1.630\end{array}$ & $\begin{array}{l}\text { Palmitic acid (16:0) } \\
\text { Linolenic acid (18:3) } \\
\text { Oleic acid (18:1) }\end{array}$ & $\begin{array}{l}671.56189 \\
649.57851 \\
645.54626\end{array}$ & $\begin{array}{l}184.1825 \\
206.1669 \\
210.1981\end{array}$ \\
\hline 6 & 18:2,18:2,16:0 & $\mathrm{C}_{55} \mathrm{H}_{98} \mathrm{O}_{6}$ & 854.73634 & 92.12 & 855.74362 & 855.74450 & -1.028 & $\begin{array}{l}599.50436 \\
575.50448\end{array}$ & $\begin{array}{l}256.24023 \\
280.24023\end{array}$ & $\begin{array}{l}256.24014 \\
280.24002\end{array}$ & $\begin{array}{l}0.351 \\
0.749\end{array}$ & $\begin{array}{l}\text { Palmitic acid (16:0) } \\
\text { Linoleic acid (18:2) }\end{array}$ & $\begin{array}{l}671.56188 \\
647.56189\end{array}$ & $\begin{array}{l}184.1826 \\
208.1826\end{array}$ \\
\hline 7 & $18: 2,18: 1,18: 2$ & $\mathrm{C}_{57} \mathrm{H}_{100} \mathrm{O}_{6}$ & 880.75199 & 90.67 & 881.75927 & 881.75821 & 1.202 & $\begin{array}{l}601.51959 \\
599.50423\end{array}$ & $\begin{array}{l}280.24023 \\
282.25588\end{array}$ & $\begin{array}{l}280.23862 \\
282.25398\end{array}$ & $\begin{array}{l}5.745 \\
6.731\end{array}$ & $\begin{array}{c}\text { Linoleic acid (18:2) } \\
\text { Oleic acid (18:1) }\end{array}$ & $\begin{array}{l}673.57718 \\
671.56171\end{array}$ & $\begin{array}{l}208.1810 \\
210.1965\end{array}$ \\
\hline 8 & $18: 3,18: 0,18: 3$ & $\mathrm{C}_{57} \mathrm{H}_{98} \mathrm{O}_{6}$ & 878.73634 & 90.44 & 879.74362 & 879.74446 & -0.955 & $\begin{array}{l}601.51975 \\
595.47226\end{array}$ & $\begin{array}{l}278.22458 \\
284.27153\end{array}$ & $\begin{array}{l}278.22471 \\
284.27220\end{array}$ & $\begin{array}{l}-0.467 \\
-2.357\end{array}$ & $\begin{array}{c}\text { Linolenic acid (18:3) } \\
\text { Stearic acid (18:0) }\end{array}$ & $\begin{array}{l}673.57741 \\
671.56184\end{array}$ & $\begin{array}{l}206.1671 \\
208.1826\end{array}$ \\
\hline 9 & 16:0,18:2,18:3 & $\mathrm{C}_{55} \mathrm{H}_{96} \mathrm{O}_{6}$ & 852.72069 & 88.06 & 853.72797 & 853.72858 & -0.715 & $\begin{array}{l}597.48851 \\
575.50425 \\
573.48877\end{array}$ & $\begin{array}{l}256.24023 \\
278.22458 \\
280.24023\end{array}$ & $\begin{array}{l}256.24007 \\
278.22433 \\
280.23981\end{array}$ & $\begin{array}{l}0.624 \\
0.899 \\
1.499\end{array}$ & $\begin{array}{l}\text { Palmitic acid (16:0) } \\
\text { Linolenic adid (18:3) } \\
\text { Linoleic acid (18:2) }\end{array}$ & $\begin{array}{l}669.54608 \\
647.56181 \\
645.54617\end{array}$ & $\begin{array}{l}184.1825 \\
206.1668 \\
208.1824\end{array}$ \\
\hline 10 & $18: 2,18: 1,18: 3$ & $\mathrm{C}_{57} \mathrm{H}_{98} \mathrm{O}_{6}$ & 878.73634 & 86.74 & 879.74362 & 879.74414 & -0.591 & $\begin{array}{l}601.51880 \\
599.50413 \\
597.48862\end{array}$ & $\begin{array}{l}278.22458 \\
280.24023 \\
282.25588\end{array}$ & $\begin{array}{l}278.22534 \\
280.24001 \\
282.25552\end{array}$ & $\begin{array}{l}-2.732 \\
0.785 \\
1.275\end{array}$ & $\begin{array}{l}\text { Linolenic acid (18:3) } \\
\text { Linoleic acid (18:2) } \\
\text { Oleic acid (18:1) }\end{array}$ & $\begin{array}{l}673.57617 \\
671.56159 \\
669.54603\end{array}$ & $\begin{array}{l}206.1680 \\
208.1826 \\
210.1981\end{array}$ \\
\hline 11 & 18:2,18:2,18:2 & $\mathrm{C}_{57} \mathrm{H}_{98} \mathrm{O}_{6}$ & 878.73634 & 85.92 & 879.74362 & 879.74414 & -0.591 & 599.50413 & 280.24023 & 280.24001 & 0.785 & Linoleic acid (18:2) & 671.56159 & 208.1826 \\
\hline 12 & $16: 0,18: 3,18: 3$ & $\mathrm{C}_{55} \mathrm{H}_{94} \mathrm{O}_{6}$ & 850.70504 & 84.33 & 851.71232 & 851.71304 & -0.845 & $\begin{array}{l}595.47294 \\
573.48877\end{array}$ & $\begin{array}{l}256.24023 \\
278.22458\end{array}$ & $\begin{array}{l}256.24010 \\
278.22427\end{array}$ & $\begin{array}{l}0.507 \\
1.114\end{array}$ & $\begin{array}{c}\text { Palmitic acid (16:0) } \\
\text { Linolenic acid (18:3) }\end{array}$ & $\begin{array}{l}667.53063 \\
645.54620\end{array}$ & $\begin{array}{l}184.1824 \\
206.1668\end{array}$ \\
\hline 13 & $18: 3,18: 1,18: 3$ & $\mathrm{C}_{57} \mathrm{H}_{96} \mathrm{O}_{6}$ & 876.72069 & 82.06 & 877.72797 & 877.72885 & -1.003 & $\begin{array}{l}599.50434 \\
595.47321\end{array}$ & $\begin{array}{l}278.22458 \\
282.25588\end{array}$ & $\begin{array}{l}278.22451 \\
282.25564\end{array}$ & $\begin{array}{l}0.252 \\
0.850\end{array}$ & $\begin{array}{l}\text { Linolenic acid (18:3) } \\
\text { Oleic acid (18:1) }\end{array}$ & $\begin{array}{l}671.56181 \\
667.53078\end{array}$ & $\begin{array}{l}206.1670 \\
210.1981\end{array}$ \\
\hline 14 & $18: 2,18: 3,18: 2$ & $\mathrm{C}_{57} \mathrm{H}_{96} \mathrm{O}_{6}$ & 876.72069 & 81.78 & 877.72797 & 877.72856 & -0.672 & $\begin{array}{l}599.50412 \\
597.48857\end{array}$ & $\begin{array}{l}278.2246 \\
280.2402\end{array}$ & $\begin{array}{l}278.2244 \\
280.2400\end{array}$ & $\begin{array}{l}0.503 \\
0.856\end{array}$ & $\begin{array}{l}\text { Linolenic acid (18:3) } \\
\text { Linoleic acid (18:2) }\end{array}$ & $\begin{array}{l}671.56144 \\
669.54599\end{array}$ & $\begin{array}{l}206.1671 \\
208.1826\end{array}$ \\
\hline 15 & 18:3,18:2,18:3 & $\mathrm{C}_{57} \mathrm{H}_{94} \mathrm{O}_{6}$ & 874.70504 & 78.77 & 875.71232 & 875.71305 & -0.834 & $\begin{array}{l}597.48864 \\
595.47313\end{array}$ & $\begin{array}{l}278.2246 \\
280.2402\end{array}$ & $\begin{array}{l}278.2244 \\
280.2399\end{array}$ & $\begin{array}{l}0.611 \\
1.106\end{array}$ & $\begin{array}{c}\text { Linolenic acid (18:3) } \\
\text { Linoleic acid (18:2) }\end{array}$ & $\begin{array}{l}669.54608 \\
667.53071\end{array}$ & $\begin{array}{l}206.1670 \\
208.1823\end{array}$ \\
\hline 16 & $18: 3,18: 3,18: 3$ & $\mathrm{C}_{57} \mathrm{H}_{92} \mathrm{O}_{6}$ & 872.68939 & 75.86 & 873.69667 & 873.69751 & -0.961 & 595.47306 & 278.2246 & 278.2245 & 0.467 & Linolenic acid (18:3) & 667.53057 & 206.1669 \\
\hline 17 & 18:2 18:2 & $\mathrm{C}_{39} \mathrm{H}_{68} \mathrm{O}_{5}$ & 616.50668 & 39.29 & 617.51395 & 617.51445 & -0.810 & 337.27418 & 280.2402 & 280.2403 & -0.143 & Linoleic acid (18:2) & - & - \\
\hline 18 & 18:3 18:2 & $\mathrm{C}_{39} \mathrm{H}_{66} \mathrm{O}_{5}$ & 614.49103 & 32.89 & 615.49830 & 615.49868 & -0.617 & $\begin{array}{l}337.27405 \\
335.25850\end{array}$ & $\begin{array}{l}278.2246 \\
280.2402\end{array}$ & $\begin{array}{l}278.2246 \\
280.2402\end{array}$ & $\begin{array}{c}-0.180 \\
0.000\end{array}$ & $\begin{array}{c}\text { Linolenic acid (18:3) } \\
\text { Linoleic acid (18:2) }\end{array}$ & - & - \\
\hline 19 & $18: 318: 3$ & $\mathrm{C}_{39} \mathrm{H}_{64} \mathrm{O}_{5}$ & 612.47538 & 27.54 & 613.48265 & 613.48300 & -0.571 & 335.25839 & 278.2246 & 278.2246 & -0.108 & Linolenic acid (18:3) & - & - \\
\hline
\end{tabular}




\subsection{Physicochemical Quality Control and Lipid Composition Profile of SIO}

The fatty acid composition in vegetable oils can change due to several reasons, such as plant phenotypic variety, plant growing conditions, type of crop nutrients, conditions of oil extraction, etc. Hence, it is practically mandatory to determine the initial physicochemical features of the sacha inchi oil. The results of the physicochemical characterization and fatty acid profile of SIO are summarized in Table 2.

Table 2. Results of physicochemical quality control and the composition of fatty acid methyl ester profiles of Sacha inchi oil.

\begin{tabular}{|c|c|c|c|}
\hline \multicolumn{2}{|c|}{ Physicochemical Parameter } & \multicolumn{2}{|l|}{ Value } \\
\hline \multicolumn{2}{|c|}{ Refractive index } & \multicolumn{2}{|l|}{1.4810} \\
\hline \multicolumn{2}{|c|}{ Saponification value $(\mathrm{mg} \mathrm{KOH} / \mathrm{g})$} & \multicolumn{2}{|l|}{251.72} \\
\hline \multicolumn{2}{|c|}{ Peroxide index $(\mathrm{meq} \mathrm{O} / \mathrm{kg})$} & \multicolumn{2}{|l|}{14.77} \\
\hline \multicolumn{2}{|c|}{ Iodine value $\left(\mathrm{g} \mathrm{I}_{2} / 100 \mathrm{~g}\right)$} & \multicolumn{2}{|l|}{195.05} \\
\hline \multicolumn{2}{|c|}{ Acid index ( $\%$ oleic acid $)$} & \multicolumn{2}{|l|}{1.31} \\
\hline \multicolumn{4}{|c|}{ Profile of lipid composition $(\% \mathrm{w} / \mathrm{w})$} \\
\hline Common name/shorthand & \multicolumn{2}{|c|}{ IUPAC Name } & Value \\
\hline Myristc acid/C14:0 & \multicolumn{2}{|c|}{ Tetradecanoic acid } & 0.02 \\
\hline Palmitic acid/C16:0 & \multicolumn{2}{|c|}{ Hexadecanoic acid } & 3.89 \\
\hline Palmitoleic acid/C16:1(n-7) & \multicolumn{2}{|c|}{ (Z)-hexadec-9-enoic acid } & 0.06 \\
\hline Heptadecanoic acid/C17:0 & \multicolumn{2}{|c|}{ Heptadecanoic acid } & 0.07 \\
\hline Heptadecanoic acid/C17:1(n-7) & \multicolumn{2}{|c|}{ (Z)-heptadec-9-enoic acid } & 0.03 \\
\hline Stearic acid/C18:0 & \multicolumn{2}{|c|}{ Octadecanoic acid } & 2.80 \\
\hline Oleic acid/C18:1(n-9) & \multicolumn{2}{|c|}{ (Z)-octadec-9-enoic acid } & 9.34 \\
\hline Linoleic acid (LA)/C18:2(n-6) & \multicolumn{2}{|c|}{ (9Z,12Z)-octadeca-9,12-dienoic acid } & 35.01 \\
\hline Linolenic acid (ALA)/C18:3(n-3) & \multicolumn{2}{|c|}{$(9 Z, 12 Z, 15 Z)$-octadeca-9,12,15-trienoic acid } & 48.39 \\
\hline Arachidic acid/C20:0 & \multicolumn{2}{|c|}{ Icosanoic acid } & 0.07 \\
\hline Gadoleic acid/C20:1(n-9) & \multicolumn{2}{|c|}{ (Z)-icos-9-enoic acid } & 0.30 \\
\hline Behenic acid/C22:0 & \multicolumn{2}{|c|}{ Docosanoic acid } & 0.02 \\
\hline
\end{tabular}

The results of refractive index, saponification value, peroxide value, iodine value, acidity index, amount of saturated fat $(6.87 \%)$, amount of monounsaturated fat $(9.73 \%)$, and amount of polyunsaturated fat (83.40\%), as well as the composition of omega-3 (48.39\%), omega-6 (35.01\%), and omega-9 (9.64\%) were very similar to the previously reported values for this oil [15]. However, it is important to note that the value obtained from the acid number (\% oleic acid) is greater than $1 \%$, and with this, such raw material does not correspond to an extra virgin oil.

\subsection{Determination of the Required HLB for SIO}

\subsubsection{Interfacial Tension Measurements}

The interfacial tension $\left(\gamma_{\mathrm{o}-\mathrm{w}}\right)$ generated in a heterogeneous system conformed by oil and water is due to several unfavorable thermodynamic phenomena, such as the incapability of the dispersing phase (water) to disintegrate the oil and generate a dispersed phase, and the incapability of the dispersing phase to generate the well-known "solvation box" [32]. In addition to the enthalpic factors just mentioned, there are also other phenomena that lead to the formation of the heterogeneous system and that correspond to the entropic factor. This factor is related to the decrease in the thermal-random motion of the water molecules, which are immediately joined to the layer of the oily phase. Such an entropic factor is the main reason for destabilization in heterodisperse systems (emulsions), because this leads to a series of dynamic events that end with the break of the system (phase separation), creating a tiny contact area known as an interface flat [33,34]. Therefore, in this assay, polysorbate 80 and sorbitan 80 surfactants were selected due to several reasons. (i) They allow obtaining different 
values of surfactant blend $\left(\mathrm{HLB}^{\mathrm{B}}\right)$. (ii) The lengths of their alkyl chains coincide with the lengths of the hydrocarbon chains of the fatty acids in SIO, favoring a better interaction between oil phase and the surfactants. (iii) At room temperature they are liquid, easing the incorporation and solubilization in the respective phases of the emulsion. Regarding the measurements of interfacial tension formed between $\mathrm{SIO}$ and ultrapure water, it was found that $\gamma_{\mathrm{o}-\mathrm{w}}$ value was $40.7 \pm 3.0 \mathrm{mN} / \mathrm{m}$. However, this value decreased with the incorporation of the surfactants in the system, where the blend of surfactants with $\mathrm{HLB}^{\mathrm{B}}$ values of 8 and 10 led to reduction in $\gamma_{\mathrm{o}-\mathrm{w}}$ value until $4.4 \pm 1.1 \mathrm{mN} / \mathrm{m}$ and $6.9 \pm 0.6 \mathrm{mN} / \mathrm{m}$, respectively, as shown in Figure 2. In this way, the change in $\gamma_{\mathrm{o}-\mathrm{w}}$ suggests that the required HLB ${ }^{\mathrm{B}}$ value to achieve the maximum stabilization of SIO is around eight.

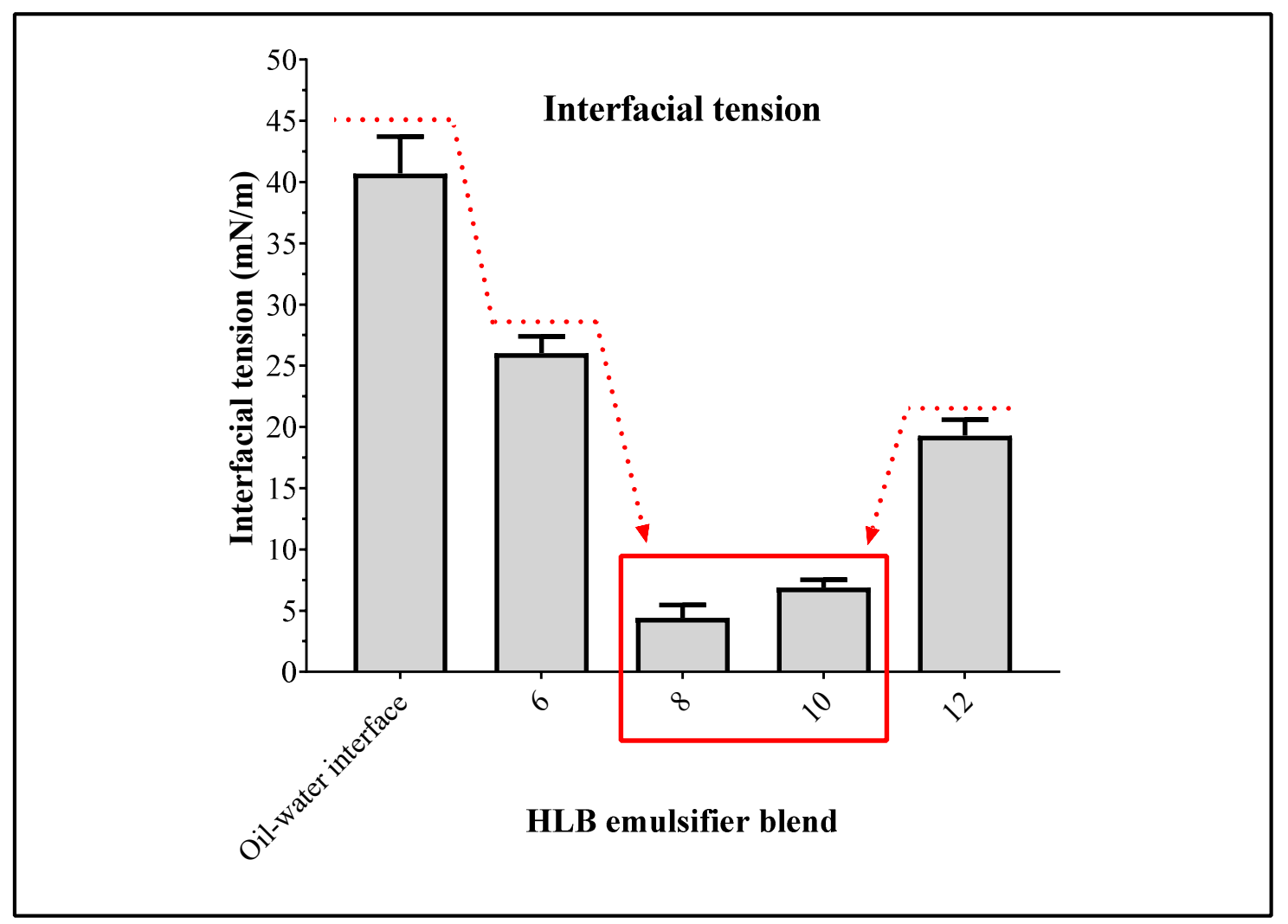

Figure 2. Change of interfacial tension formed between sacha inchi oil and ultra-pure water at different values of HLB surfactants blend.

On the other hand, the results corresponding to physicochemical characterization of the emulsion made up with SIO are depicted in Figure 3. 


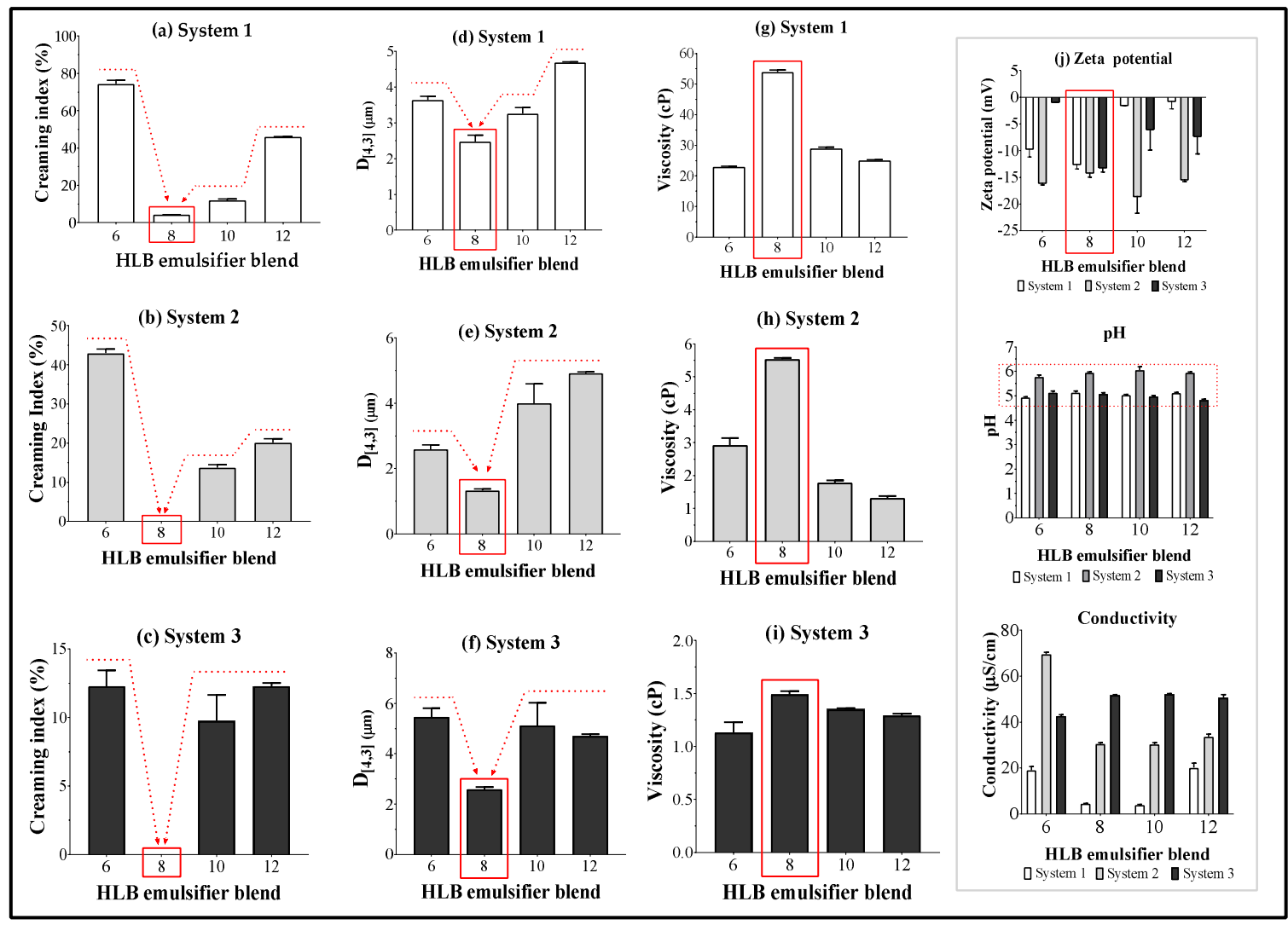

Figure 3. Physicochemical characterization of oil-in-water emulsions elaborated with SIO at different values of HLB surfactants blend, where system 1 corresponds to steareth 2 + steareth 20, system 2 corresponds to glyceryl stearate + polyoxyl 40 stearate and system 3 corresponds to sorbitan oleate + polysorbate 80 .

\subsubsection{Creaming Index and Droplet Size}

The first aspect to highlight in this study is the low amount of surfactant blend used in the emulsified formulations $(2 \% \mathrm{w} / \mathrm{w})$, and therefore, it is possible to guarantee that the stabilization effect in the heterodisperse systems is provided exclusively by the capability of the surfactants to reduce the interface tension and not due to other effects, like micellar stabilization or rheological stabilization.

With respect to the results of $\mathrm{CI}$ at zero-time, it was found that surfactant blends with $\mathrm{HLB}^{\mathrm{B}}=8$ always cause the lowest IC (Figure 3a-c), reaching values of $4.3 \pm 0.1$ for system 1 and 0 for systems 2 and 3. These results are very consistent with those previously obtained in the interfacial tension assays, as well as those found by Kiattiphumi and Ampa [16], who reported that the HLB $^{B}$ value that leads to the maximum stabilization of oil-in-water emulsions made with SIO was 8.5. However, it should be mentioned that in such a study, they evaluated different conditions than those employed by us, where they used a lower proportion oil $(5 \% \mathrm{w} / \mathrm{w})$, a higher concentration of surfactant blend $(5 \% \mathrm{w} / \mathrm{w})$, and a thickening agent as a co-stabilizer.

In regard to the droplet size data (Figure $3 \mathrm{~d}-\mathrm{f}$ ), it was found that these results also show a similar behavior to that described in the interfacial tension and CI assays, where the lowest droplet size values (between $1.3 \mu \mathrm{m}$ and $2.6 \mu \mathrm{m}$ ) were reached at $\mathrm{HLB}^{\mathrm{B}}=$ 8.0. Such particle sizes also suggest that the dispersed oil droplets are well stabilized, quite possibly by the formation of a compact layer of surfactants at the interface.

\subsubsection{Viscosity Assay}

The results of the viscosity assay also agree with those results previously obtained in the studies of interfacial tension, $\mathrm{CI}$, and droplet size, where the maximum viscosity values were achieved 
when the $\mathrm{HLB}^{\mathrm{B}}=8$ (Figure $3 \mathrm{~g}-\mathrm{i}$ ). This behavior could be explained, considering the formation of compact oil droplets very well stabilized, which interact in situ, with other stabilized droplets, where the polyethoxylated groups of the hydrophilic surfactants (steareth 20, polyoxyl-40-stearate, and polysorbate 80 ) form hydrogen bonds that increase the cohesiveness in the system, and thus, the viscosity. On the other hand, the differences observed in viscosity values for the emulsified systems could be explained according to the physicochemical features of the surfactants employed. In the case of system 1, which showed the higher viscosity value ( $\sim 54.0 \mathrm{Cp})$ followed by system $2(\sim 5.5 \mathrm{Cp})$, the surfactant used are waxes at room temperature, and thus, during the emulsification process and specifically the cooling step, those surfactants solidify, forming interfacial films that are very compact and viscous [35]. On the contrary, system 3 showed the lowest viscosity value (1.5 cp), where the surfactants used are liquid, which form less compact interfacial layers.

\subsubsection{Zeta Potential, Ph, and Conductivity}

The results of zeta potential (Figure $3 \mathbf{j}$ ) are very interesting if we consider that all the surfactants used in the study were neutral, and therefore, the zeta potential values should be very close to zero. However, all the zeta potential values obtained were negative. Such behavior can be explained due to the spontaneous formation of a tiny monolayer of hydroxyl ions located in the interface oil-surfactant-water and which come from the autoprotolysis of water [36,37]. Regarding the electrical conductivity values, they can be attributed to the presence of hydronium and hydroxyl ions from the autoprotolysis of water, as well as the ions from the ionization of carbonic acid present in the medium. Although there is not a tendency between the conductivity and the $\operatorname{HLB}^{\mathrm{B}}$ values, it can be possible to note that system 1 has the lowest conductivity values $(<20 \mu \mathrm{S} / \mathrm{m})$, while system 3 has the highest conductivity values $(>40 \mu \mathrm{S} / \mathrm{m})$. These results are very consistent, considering that at high viscosity there is low ionic mobility, and thus, system 1 has high viscosity and low conductivity, while system 3 is the opposite. In contrast, the $\mathrm{pH}$ characterization of emulsified systems always described slightly acidic values ( $\mathrm{pH}=5-6)$. Such a result is attributed to the possible incorporation of $\mathrm{CO}_{2}$ (gas) into the emulsions during the emulsification process, where such gas is subsequently transformed into carbonic acid.

\subsection{Thermal Stability Assay of Sacha Inchi Emulsion}

Thermal stress stability studies are very important in the primary stages of formulation or evaluation of prototypes (product pre-formulation), because they provide information in a very short time (1 month) about the possible physical, chemical, and microbiological changes that the system could undergo [38]. For this study, the thermal stability analysis was addressed only to establish the physical changes in the emulsified system, and therefore corroborate if the required HLB values for the SIO found at zero time remained constant or varied. For this, the first parameter analyzed was the $\mathrm{CI}$, which is strongly related to the final stage of physical destabilization for a heterodisperse system (emulsion breaking) [39]. In the case of CI values at time zero for the system 1 , it was found that the lowest value of such a parameter $(\mathrm{CI}=4.3 \pm 0.1)$ was reached when the emulsions had a $\mathrm{HLB}^{\mathrm{B}}$ of eight (Figure 3a). However, with time, these systems start to separate phases after the second week and only the formulations with a $\mathrm{HLB}^{\mathrm{B}}$ value of ten remained stable. Hence, the data reported for system 1 corresponds to the emulsion with a value of $\mathrm{HLB}^{\mathrm{B}}$ of ten. On the other hand, the emulsions of systems 2 and 3 were stable only at values of $\mathrm{HLB}^{\mathrm{B}}$ of eight. In general, physical changes were observed changes regarding $\mathrm{CI}$, droplet size, viscosity, zeta potential, $\mathrm{pH}$, and electrical conductivity for all the emulsified systems from the first week. These changes are shown in Figure 4. 


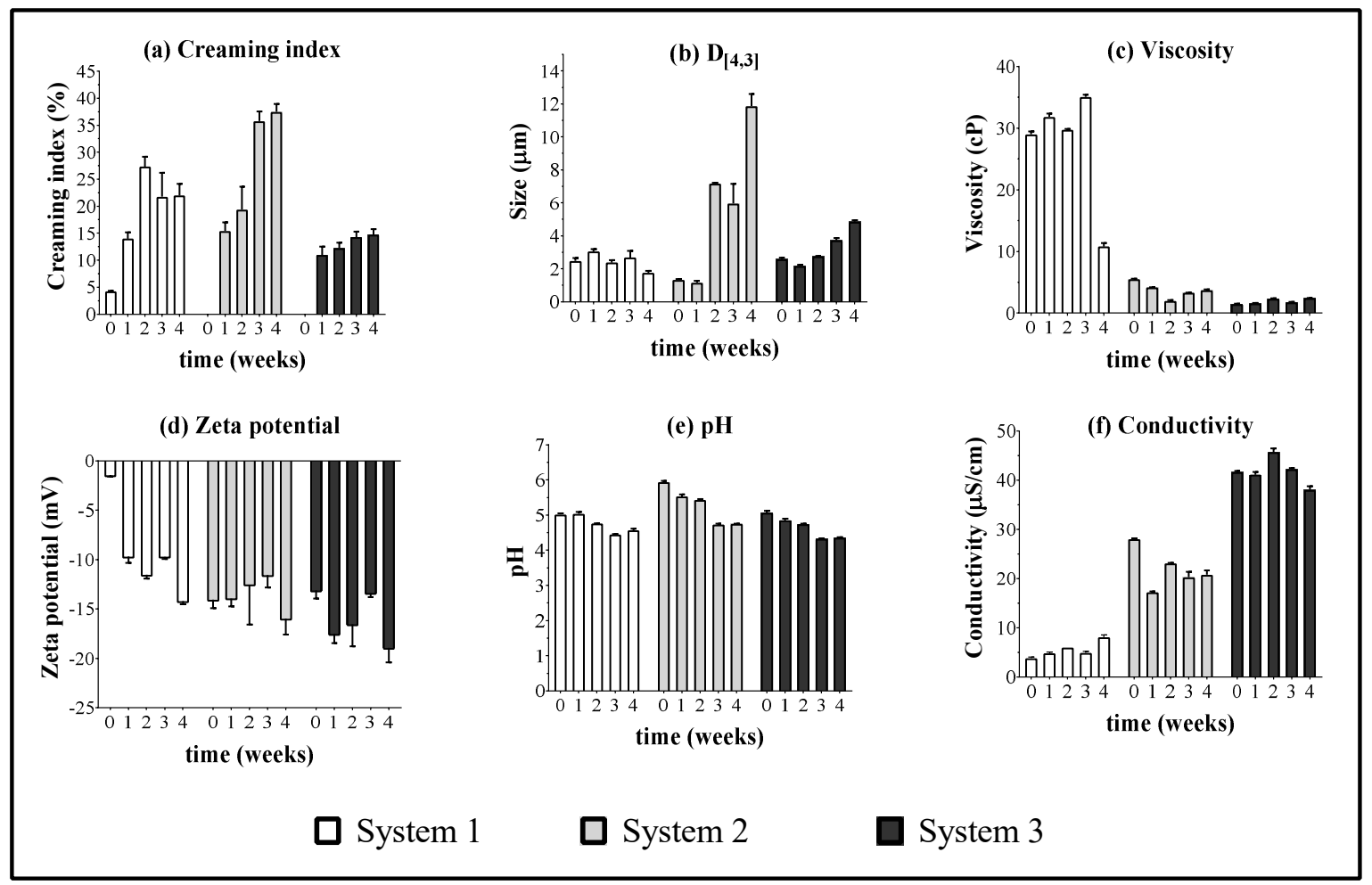

Figure 4. Physicochemical characterization of oil-in-water emulsions to thermal stress conditions. The data of system 1 corresponds to the formulations with a HLBB of ten, whilst the systems 2 and 3 correspond to the formulations with HLBB of eight.

According to system 1, which had steareth 2 and steareth 20 as surfactant blend, it was found that these provided the least capability to stabilize the emulsified formulations of sacha inchi and the results obtained did not depend only on interfacial phenomena $\left(\mathrm{HLB}^{\mathrm{B}}\right)$, but also on other factors such as rheological stabilization. On the contrary, systems 2 and 3 show a better capability to stabilize the emulsions. In relation to system 2, which had surfactants glyceryl stearate and polyoxyl 40 stearate, we appreciated an appropriate stabilization of the emulsions. However, after the second week, the CI values increased, as well as the droplet size. Regarding system 3, it proved to be the most effective to stabilize the emulsified systems, due to the lower CI values obtained and slight changes in droplet size; even so, this system had the lowest viscosity values. This result can be explained considering that sorbitan 80 and polysorbate 80 surfactants have alkyl chains quite similar to those present in the fatty acids in SIO. Also, such surfactants are easier to incorporate in the respective emulsion phases than the rest of the surfactants evaluated. For the results of zeta potential and conductivity, there were no marked changes with respect to study time. On the other hand, no trend was observed between the $\mathrm{pH}$ and the time of the thermal stability assay, where such parameters remained constant between 5 and 6, which is explained by the possible formation of carbonic acid, as described above.

\section{Conclusions}

The physicochemical characterization of the emulsions prepared with SIO showed values very similar to those previously reported for this oil. Likewise, it was found that the composition of fatty acids, and especially the omega-3 (48.39\%), omega-6 (35.01\%), and omega-9 $(9.64 \%)$, also coincides with the previous description given for other sacha inchi oil (volubilis variety).

It was also observed that the maximum decrease of the interfacial tension between SIO and ultrapure water was achieved, when the surfactant blend has a value of $\mathrm{HLB}^{\mathrm{B}}$ of eight. Equally, it was found that at such $\mathrm{HLB}^{\mathrm{B}}$ value, the maximum stabilization of the emulsions is reached. In relation to the different mixtures of surfactants used, the blend of sorbitan 80 and polysorbate 80 proved to be the most 
effective to stabilize emulsions elaborated with sacha inchi. The fatty acid composition of SIO displayed a high profile of unsaturated fatty acid, in which $50 \%$ of them are arranged in TAG13 (18:3,18:1,18:3), TAG14 (18:2,18:3,18:2), TAG15 (18:3,18:2,18:3), and TAG16 (18:3,18:3,18:3). Diacylglycerides comprise close to $8 \%$ of relative abundance in SIO. Due the complexity of analyzed TAG and DAG using conventional techniques, high-resolution mass spectrometry with APCI ion source turns out to be an excellent analytical tool, not only for chemical characterization, but also for quality control during extraction of raw material and monitoring throughout further processing.

On the other hand, the physical-chemical characterization of the oil and emulsions provided a starting point for advance formulation for several industries like cosmetic, pharmaceutical, and foodstuff. Keeping in mind the exceptional nutritional features, this oil will be undeniably converted in the next decades into input for new product formulations. Also, seeing the tremendous impact of illicit crop replacement over the conflict in Colombia, and at the same time bearing in mind its quality, the authors encourage the Colombian government to extend and support the conditions to provide peasants and remote provinces located in the conflict area to establish a new and highly prolific economic chain from the vast and fruitful agriculture that the country has. The most remote regions (Putumayo, Cauca, Nariño, and Caquetá departments) have the greatest and most extraordinary ground conditions for its culture, which makes it a successful and strategical decision.

Supplementary Materials: The following are available online at http://www.mdpi.com/2079-9284/6/4/70/s1. Table S1: Formulations of oil-in-water emulsion made with SIO. All (\%) corresponding to \% weight/weight; Table S2: Values of shear rate applied in the emulsion and nano-emulsion systems in viscometric assay; Figure S1: High-resolution tandem mass spectra of SIO Glycerolipids:

Author Contributions: Methodology and investigation: D.P.-C., V.D., C.M., D.M.P., S.K.R.-A.; Supervision writing-review and editing: O.S.-C., U.H., G.M., and C.H.S.

Funding: This research was funded the internal grant of Icesi University No. CA CA041368.

Acknowledgments: The authors thank the Universidad Icesi for financial resources in the execution of this research, and Nutresacha S.A. for providing the SIO used in this study.

Conflicts of Interest: The authors declare no conflict of interests.

\section{References}

1. Follegatti-Romero, L.A.; Piantino, C.R.; Grimaldi, R.; Cabral, F.A. Supercritical $\mathrm{CO}_{2}$ extraction of omega-3 rich oil from Sacha inchi (Plukenetia volubilis L.) seeds. J. Supercrit. Fluids 2009, 49, 323-329. [CrossRef]

2. Marris, E. Amazon plant discovery could yield green cash crop. Nature 2013. [CrossRef]

3. Wang, S.; Zhu, F.; Kakuda, Y. Sacha inchi (Plukenetia volubilis L.): Nutritional composition, biological activity, and uses. Food Chem. 2018, 265, 316-328. [CrossRef]

4. Fanali, C.; Dugo, L.; Cacciola, F.; Beccaria, M.; Grasso, S.; Dachà, M.; Dugo, P.; Mondello, L. Chemical characterization of Sacha inchi (Plukenetia volubilis L.) oil. J. Agric. Food Chem. 2011, 59, 13043-13049. [CrossRef]

5. Calabriso, N.; Scoditti, E.; Pellegrino, M.; Carluccio, M.A. Olive oil. In The Mediterranean Diet: An Evidence-Based Approach; Academic Press: Cambridge, MA, USA, 2014; ISBN 9780124079427.

6. Bährle-Rapp, M. Coconut oil. In Springer Lexikon Kosmetik und Körperpflege; Springer: Berlin/Heidelberg, Germany, 2010.

7. Maleš, Ž.; Mišković, G.; Bojić, M.; Ćurak, I. Argan oil. Farm. Glas. 2018, 74, 817-824.

8. Duarte, P.F.; Chaves, M.A.; Borges, C.D.; Mendonça, C.R.B. Avocado: Characteristics, health benefits, and uses. Int. News Fats Oils Relat. Mater. 2016, 46, 747-754. [CrossRef]

9. Gülçin, I.; Elmastaş, M.; Aboul-Enein, H.Y. Antioxidant activity of clove oil-A powerful antioxidant source. Arab. J. Chem. 2012, 5, 489-499. [CrossRef]

10. Juliano, C.; Magrini, G. Cosmetic functional ingredients from botanical sources for anti-pollution skincare products. Cosmetics 2018, 5, 19. [CrossRef]

11. Fonseca-Santos, B.; Corrêa, M.A.; Chorilli, M. Sustainability, natural and organic cosmetics: Consumer, products, efficacy, toxicological and regulatory considerations. Braz. J. Pharm. Sci. 2015, 51, 17-26. [CrossRef]

12. Matić, M.; Puh, B. Consumers'purchase intentions towards natural cosmetics. Ekon. Vjesn. 2016, $29,53-64$. 
13. Moure, A.; Cruz, J.M.; Franco, D.; Domínguez, J.M.; Sineiro, J.; Domínguez, H.; Núñez, M.J.; Parajó, J.C. Natural antioxidants from residual sources. Food Chem. 2001, 72, 145-171. [CrossRef]

14. Gonzales, G.F.; Gonzales, C.; Villegas, L. Exposure of fatty acids after a single oral administration of sacha inchi (Plukenetia volubilis L.) and sunflower oil in human adult subjects. Toxicol. Mech. Methods 2014, 24, 60-69. [CrossRef]

15. Vicente, J.; de Carvalho, M.G.; Garcia-Rojas, E.E. Fatty acids profile of sacha inchi oil and blends by 1 H NMR and GC-FID. Food Chem. 2015, 181, 215-221. [CrossRef]

16. Saengsorn, K.; Jimtaisong, A. Determination of hydrophilic-lipophilic balance value and emulsion properties of sacha inchi oil. Asian Pac. J. Trop. Biomed. 2017, 7, 1092-1096. [CrossRef]

17. Tunkam, P.; Satirapipathkul, C. Preparation of nanoemulsion from sacha inchi oil/water by emulsion phase inversion methods. Key Eng. Mater. 2016, 675, 57-60. [CrossRef]

18. Silva, K.F.C.E.; da Silva Carvalho, A.G.; Rabelo, R.S.; Hubinger, M.D. Sacha inchi oil encapsulation: Emulsion and alginate beads characterization. Food Bioprod. Process. 2019, 116, 118-129. [CrossRef]

19. Sanchez-Reinoso, Z.; Gutiérrez, L.F. Effects of the emulsion composition on the physical properties and oxidative stability of sacha inchi (Plukenetia volubilis L.) oil microcapsules produced by spray drying. Food Bioprocess Technol. 2017, 10, 1354-1366. [CrossRef]

20. Vicente, J.; Pereira, L.J.B.; Garcia-Rojas, E.E.; de Souza Cezarino, T.; de Carvalho, M.G.; da Rocha, E.P.; Sá, G.R.; Gamallo, O.D. Microencapsulation of sacha inchi oil using emulsion-based delivery systems. Food Res. Int. 2017, 99, 612-622. [CrossRef]

21. Fernandes, C.P.; Mascarenhas, M.P.; Zibetti, F.M.; Lima, B.G.; Oliveira, R.P.R.F.; Rocha, L.; Falcão, D.Q. HLB value, an important parameter for the development of essential oil phytopharmaceuticals. Braz. J. Pharmacogn. 2013, 23, 108-114. [CrossRef]

22. Schmidts, T.; Schlupp, P.; Gross, A.; Dobler, D.; Runkel, F. Required HLB determination of some pharmaceutical oils in submicron emulsions. J. Dispers. Sci. Technol. 2012, 33, 816-820. [CrossRef]

23. American Oil Chemists' Society. Official Methods and Recommended Practices of the AOCS; American Oil Chemists' Society: Champaign, IL, USA, 2017.

24. U.S. Pharmacopoeia. USP <191> Identification Tests-General; U.S. Pharmacopoeia: Rockville, MD, USA, 2018.

25. Kahl, H.; Wadewitz, T.; Winkelmann, J. Surface tension of pure liquids and binary liquid mixtures. J. Chem. Eng. Data 2003, 48, 580-586. [CrossRef]

26. Barth, H.G.; Sun, S.T. Particle size analysis. Anal. Chem. 1989, 61, 143-152. [CrossRef]

27. Amziane, S.; Collet, F.; Lawrence, M.; Magniont, C.; Picandet, V.; Sonebi, M. Recommendation of the RILEM TC 236-BBM: Characterization testing of hemp shiv to determine the initial water content, water absorption, dry density, particle size distribution and thermal conductivity. Mater. Struct. 2017, 50. [CrossRef]

28. Picandet, V. Particle size distribution. In RILEM State-of-the-Art Reports; Springer: Dordrecht, The Netherlands, 2017.

29. Hsu, F.F.; Turk, J. Electrospray ionization multiple-stage linear ion-trap mass spectrometry for structural elucidation of triacylglycerols: Assignment of fatty acyl groups on the glycerol backbone and location of double bonds. J. Am. Soc. Mass Spectrom. 2010, 21, 657-669. [CrossRef]

30. Xu, Y.; Brenna, J.T. Atmospheric pressure covalent adduct chemical ionization tandem mass spectrometry for double bond localization in monoene-and diene-containing triacylglycerols. Anal. Chem. 2007, 79, 2525-2536. [CrossRef]

31. Murphy, R.C.; Leiker, T.J.; Barkley, R.M. Glycerolipid and cholesterol ester analyses in biological samples by mass spectrometry. Biochim. Biophys. Acta Mol. Cell Biol. Lipids 2011, 1811, 776-783. [CrossRef]

32. Blees, M.H. Foundations of colloid science. Colloids Surfaces A Physicochem. Eng. Asp. 2002, $210,125$. [CrossRef]

33. Wang, S.; Zhang, Y.; Abidi, N.; Cabrales, L. Wettability and surface free energy of graphene films. Langmuir 2009, 25, 11078-11081. [CrossRef]

34. Silverstein, T.P. The real reason why oil and water don't mix. J. Chem. Educ. 1998, 75, 116. [CrossRef]

35. Avranas, A.; Stalidis, G. Interfacial properties and stability of oil-in-water emulsions stabilized with binary mixtures of surfactants. J. Colloid Interface Sci. 1991, 143, 180-187. [CrossRef]

36. Hunter, R.J. Zeta Potential in Colloid Science: Principles and Applications; Academic Press: Cambridge, MA, USA, 2013; ISBN 9781483214085. 
37. Wiacek, A.; Chibowski, E. Zeta potential, effective diameter and multimodal size distribution in oil/water emulsion. Colloids Surfaces A Physicochem. Eng. Asp. 1999, 159, 253-261. [CrossRef]

38. Han, J.; Davis, S.S.; Washington, C. Physical properties and stability of two emulsion formulations of propofol. Int. J. Pharm. 2001, 215, 207-220. [CrossRef]

39. French, D.J.; Taylor, P.; Fowler, J.; Clegg, P.S. Making and breaking bridges in a Pickering emulsion. J. Colloid Interface Sci. 2015, 441, 30-38. [CrossRef]

(C) 2019 by the authors. Licensee MDPI, Basel, Switzerland. This article is an open access article distributed under the terms and conditions of the Creative Commons Attribution (CC BY) license (http://creativecommons.org/licenses/by/4.0/). 